\title{
First-Principles Studies of Local Order in Relaxor Ferroelectrics ${ }^{1}$
}

\author{
Mark Wensell and Henry Krakauer \\ College of William and Mary ${ }^{2}$, Department of Physics \\ P.O. Box 8795, Williamsburg, VA 23187-8795
}

\begin{abstract}
A key to optimizing the growth of the new single-crystal relaxor ferroelectrics [1] is resolving basic questions concerning their structural properties and energetics. We report on initial first-principles total energy and force calculations, examining the energetics of local order in PZN type relaxors.
\end{abstract}

\section{INTRODUCTION}

The optimization of the electromechanical properties of the new single-crystal relaxor ferroelectrics [1] and the challenge of growing large crystals raise several fundamental questions. What is the structure of these materials, and how is it related to their properties? We first briefly review the present understanding of these questions and the capabilities of current theoretical approaches in addressing them.

Although some structural features of relaxor ferroelectrics are understood, their precise microscopic structure is unknown at the present time. (This is, of course, even more true of the new single crystal relaxor ferroelectrics.) The task of determining the atomic geometry is complicated by the long-range disorder inherent in relaxor ferroelectrics and the infinite-range Coulomb interaction between ions, which imposes severe constraints on the structure. It is perhaps not surprising then that despite years of extensive effort there are many fundamental questions about the atomic geometry of relaxors that are still unsettled.

For example, in PMN type relaxors with the stoichiometry $\mathrm{Pb}\left(\mathrm{B}_{1 / 3}^{2+} \mathrm{B}_{2 / 3}^{5+}\right) \mathrm{O}_{3}$ (here $\left.\mathrm{B}^{2+}=\mathrm{Mg}, \mathrm{Co}, \mathrm{Ni}, \mathrm{Zn} ; \mathrm{B}^{5+}=\mathrm{Nb}, \mathrm{Ta}\right)$, the B-sites must accommodate a 2:1 distribution of cations with very different positive charges. Experimental evidence indicates that PMN crystals do this by forming 1:1 ordered nanometer scale domains dispersed in a disordered matrix. [4-9] The ordered domains, probed by diffraction

1) To be published in the AIP proceedings of the Fifth Williamsburg Workshop on First-Principles Calculations for Ferroelectrics, February 1998.

2) Supported by the Office of Naval Research grant N00014-97-1-0049. 
and high-resolution TEM studies, are seen to have a 1:1 distribution of two different cation sites (designated $\beta^{\prime}$ and $\beta^{\prime \prime}$ ) arranged on a face-centered $\mathrm{NaCl}$-type lattice with a doubled perovskite repeat along the [111] direction. This much seems to be generally accepted. Other details about the structure are considerably less certain, as discussed below, with several basic questions that are still not settled: 1) Which atoms reside on $\beta^{\prime}$ and $\beta^{\prime \prime}$ sites? 2) Are the 1:1 nano-domains intrinsically size-limited by the Coulomb interaction or are they the result of particular crystal growth and annealing procedures? It is evident that the $\beta^{\prime}$ and $\beta^{\prime \prime}$ site assignments play a crucial role in answering the second question. For if the nano-domains are not charge-neutral, Coulomb interactions would be expected to limit the growth of these domains.

One answer to these questions is provided by the widely accepted space-charge model, which relates the observed relaxor behavior to postulated nanometer scale concentration inhomogeneities. $[2,4,10,11]$ The space-charge model postulates that the nano-domains are $\mathrm{B}^{5+}$ - deficient, with the $\beta^{\prime}$ and $\beta^{\prime \prime}$ sites occupied exclusively by the $\mathrm{B}^{2+}$ and $\mathrm{B}^{5+}$ cations respectively, in a $\mathrm{NaCl}$ like structure. The ordered regions thereby carry a net negative charge. This charge imbalance is then compensated by a $\mathrm{B}^{5+}$-rich disordered matrix. The resulting large self-Coulomb repulsion of the charged regions would be expected to limit the size of the nano-domains, and the random distribution of nano-domain polarizations is an appealing explanation for the observed diffuse and frequency dependent permitivity of the relaxor ferroelectrics. Support for this model includes an observed lack of domain coarsening with long-term annealing in all but the most recent experiments, discussed below. This has contributed to widespread acceptance of the space-charge model, despite the absence of direct evidence for this type of nano-level compositional segregation. $[2,9]$

Recently, however, Akbas and Davies have reported experiments on $\mathrm{Pb}\left(\mathrm{Mg}_{1 / 3} \mathrm{Ta}_{2 / 3}\right) \mathrm{O}_{3}-\mathrm{PbZrO}_{3}$, in which the size of fully chemically ordered 1:1 domains (evidenced by x-ray diffraction) were increased by two orders of magnitude through annealing conducted at $1325{ }^{\circ} \mathrm{C}$; moreover, fully ordered ceramics comprised of large domains were found to retain relaxor behavior. [2] (Previous annealing was restricted to below $970{ }^{\circ} \mathrm{C}$.) The concentration inhomogeneities of the space-charge model are inconsistent with these recent results, since Coulomb repulsion would limit the size of the $\mathrm{B}^{5+}$-deficient 1:1 domains. There are several important implications of this recent experiment. First, the distribution of $\mathrm{B}^{2+}$ and $\mathrm{B}^{5+}$ concentrations must be homogeneous at the atomistic scale to permit the growth of large 1:1 domains. Second, the retention of relaxor behavior of the fully ordered ceramics suggests that the relaxor behavior is due to random local (at the atomistic scale) polarization fluctuations. Akbas and Davies proposed a previously dismissed charge-balanced random-site model for cation ordering as a possible candidate for atomistic scale homogeneity. According to this scheme, the $\beta^{\prime \prime}$ sites are occupied exclusively by $\mathrm{B}^{5+}$, while the $\beta^{\prime}$ sites are occupied by a $50-50$ random mixture of $\mathrm{B}^{2+}$ and $\mathrm{B}^{5+}$ ions. The structural formula can be represented 
as $\mathrm{Pb}\left[\mathrm{B}_{2 / 3}^{2+} \mathrm{B}_{1 / 3}^{5+}\right]_{1 / 2}\left[\mathrm{~B}_{1 / 2}^{5+}\right] \mathrm{O}_{3}$. The random-site model is consistent with the domain coarsening observed in this experiment.

It is also consistent with the observations of Teslic et al. [3] using pulsed neutron atomic pair-distribution function measurements of PMN, PZ and PZT, which show that the atomic environments, particularly that of $\mathrm{Pb}$, are similar in all these compounds. Teslic et al. suggest that a large portion of the ferroelectric polarization is provided by $\mathrm{Pb}$ displacements (to accommodate the lone-pair electrons), and these displacements are closely coupled to the rotation of the $\mathrm{BO}_{6}$ octahedra. This picture provides another possible framework for understanding relaxor behavior at the atomic level. Since the coupling of the $\mathrm{Pb}$ polarization to the environment and the ease of rotation of the $\mathrm{BO}_{6}$ will have different energy scales, the random distribution of the $\mathrm{B}$ cations on the $\beta^{\prime}$ sites might be expected to lead to relaxor behavior.

The above illustrates the status and shortcomings in the present understanding of the geometry of even the conventional relaxor ferroelectrics. Even less is known about the single crystal relaxors. Yet knowledge of the atomic geometry is certainly a necessary prerequisite for understanding and optimizing their remarkable properties. A complete description of the atomic geometry will have to resolve questions like the space-charge versus random-site models. To do this, local effects such as atomic relaxation (i.e. $\mathrm{Pb}$ displacements and $\mathrm{BO}_{6}$ octahedra rotations) away from ideal perovskite structure will also have to be treated. Such short-range order likely plays a crucial role in defining the properties and the relative stability of different structures. If the atomistically homogeneous models of the relaxors are in fact correct, then this is likely to be the case, because in this scenario the nano-domains are not intrinsically limited in size by Coulomb effects, but inhibited in growth by geometrical frustration, similar to spin glasses. [3] Indeed, the role of $\mathrm{PbTiO}_{3}$ admixture in PMN-PT single-crystal relaxors may be to reduce frustration and facilitate single-crystal growth.

A powerful theoretical approach to characterize the short-range order is to use first-principles calculations to determine local atomistic structures and resolve the energetics of the space-charge versus random-site model. In this initial study, we employ supercells containing 15 - 30 atoms to model the short-range order. To efficiently handle the computational burden incurred in such large scale calculations, we employ our first-principles mixed-basis projector method. [12,13]

Looking ahead, we eventually want to model growth processes as well as longrange disorder and domain interactions. A direct first-principles approach, however, would require very large simulation cells, a task that exceeds the capability of any presently available first-principles method. Nevertheless, complementary methods have been developed to extend the reach of the first-principles methods. One such method is based on using first-principles derived effective Hamiltonians. These Hamiltonians act in a reduced sub-space of the full Hilbert space, retaining only the most important degrees of freedom.

Molecular dynamics and Monte Carlo simulations with such effective Hamiltonians have demonstrated their ability to successfully predict phase transitions and 
temperature-dependent static and dynamic properties for the pure constituents systems like $\mathrm{BaTiO}_{3}, \mathrm{KNbO}_{3}$, and $\mathrm{PbTiO}_{3}$. [14,15] Thus, the correct phase sequence of ferroelectric phase transitions was obtained in these materials, showing that the first-principles effective Hamiltonian used in the simulations captures the essential behavior of the microscopic fluctuations driving the transitions. In $\mathrm{KNbO}_{3}$ and $\mathrm{BaTiO}_{3}$ molecular dynamics simulations have revealed the existence of localized preformed dynamic chain-like structures, which are present even in the hightemperature paraelectric phase, well above the cubic-tetragonal phase transition. [15] The molecular dynamics simulations also reproduced the essential features of the diffuse x-ray scattering measurements and the weak temperature dependence of measured diffuse streak patterns. These studies provided a framework for understanding both the displacive and order-disorder characteristics of these phase transitions as both arising from the softening of an entire branch of unstable transverse-optic phonon modes.

We hope that the present calculations for PZN can lay the foundation for extending such finite temperature studies to the relaxor ferroelectrics. Extending the effective Hamiltonian approach to alloy systems presents many challenges, and may ultimately be unsuccessful. In that case other approaches, such as effective interatomic potentials or generalized shell models may be better suited to the problem. In any case, any of these complimentary methods will be based on first-principles results of the type presented below.

\section{METHODOLOGY}

Self-consistent local density functional approximation (LDA) calculations were carried out using a first-principles mixed basis projector method. [12] The method uses a Kerker type pseudopotential to remove the chemically inert innermost coreelectron states from the Hilbert space. For the 30-atom supercells, there were 134 occupied bands, and the basis set consisted of about 3600 plane waves, corresponding to about a 19 Ry kinetic energy cut-off. This relatively low kinetic energy cut-off is achieved by the inclusion of 148 local basis functions, including $\mathrm{Pb} 5 d, \mathrm{Zn} 3 d, \mathrm{Nb}$ $4 s, 4 p, 4 d$, and $\mathrm{O} 2 s, 2 p$ orbitals. The semicore $\mathrm{Nb} 4 s$ and $4 p$ states were retained in the variational basis, due to their spatially extended character. The method benefits from Car-Parinello CPU-time scaling, facilitating the treatment of these large supercells. It achieves this using fast Fourier transforms and the fact that the local orbitals are strictly confined within non-overlapping muffin-tin spheres. Thus there are no two- or three-center integrals, and the local orbitals overlap only with the plane waves. The muffin-tin sphere radii were 2.0, 1.9, 1.6, and 1.65 a.u. for $\mathrm{Pb}, \mathrm{Zn}, \mathrm{Nb}$, and $\mathrm{O}$ atoms, respectively. In these initial calculations, we chose the volume to correspond to the experimentally observed volume of $\mathrm{PbTiO}_{3}$, but allowed the internal atomic positions to relax to the lowest energy. Calculations were carried out for 30- and 15-atom supercells, using one and two k-points, respectively. This corresponds, roughly, to a 2x2x2 Monkhorst-Pack mesh. 


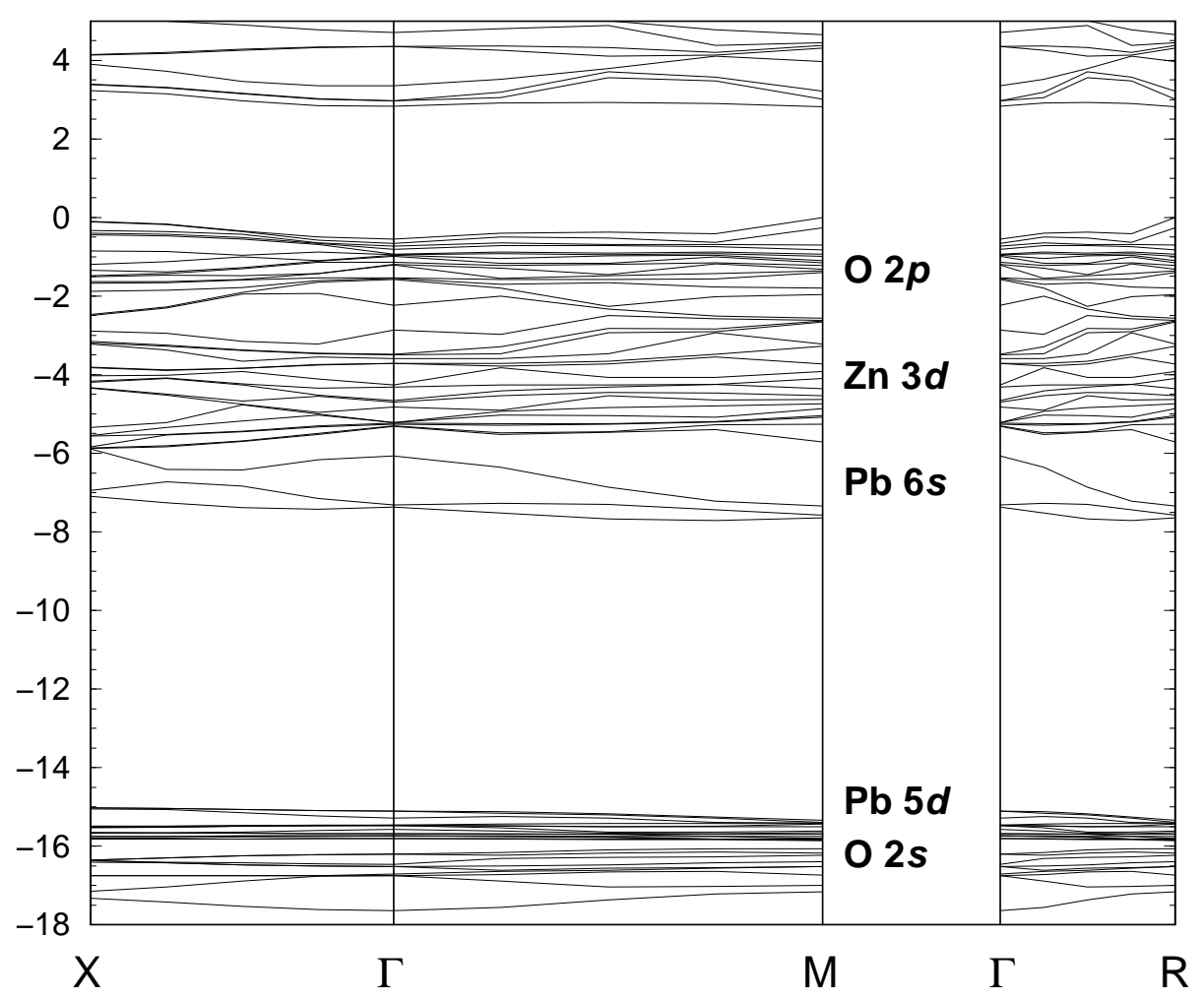

FIGURE 1. Band structure of 15 atom "3spcchrg" cell

\section{RESULTS AND DISCUSSION}

We performed calculations on five different structural models, based on [111] B cation-plane stacking. Two are based on [111] planes containing either Zn or Nb. The first is a 30 atom model that has the stacking sequence $\mathrm{Zn}, \mathrm{Nb}, \mathrm{Zn}, \mathrm{Nb}, \mathrm{Nb}$, $\mathrm{Nb}, \mathrm{Zn} \ldots$, and we refer to this model as "6spcchrg", containing 6 perovskite units. It features the 1:1 ordering proposed by the space charge model and in addition, crudely simulates a Nb rich region. The second is a 15 atom model, "3spcchrg", with the stacking sequence $\mathrm{Zn}, \mathrm{Nb}, \mathrm{Nb} \ldots$... We have imposed $R 3 m$ space group symmetry on both of these models.

In the remaining three models the [111] B-site planes do not all contain pure $\mathrm{Zn}$ or $\mathrm{Nb}$. There are two 30 atom models, each of which is based on the stacking sequence $\beta^{\prime} \beta^{\prime \prime} \ldots$, where $\beta^{\prime}$ is $\left[\mathrm{Zn}_{2 / 3} \mathrm{Nb}_{1 / 3}\right]_{1 / 2}$ and the $\beta^{\prime \prime}$ site is pure $\mathrm{Nb}$. These two differ by the in-plane lattice vectors and have different symmetry. One of these, "6tria", has symmetry Imm2 and is based on a triangular arrangement of the $\mathrm{Zn}$ and Nb atoms. The other, "6lin", has symmetry Fmm2 and features adjacent rows of $\mathrm{Zn}$ atoms separating rows of $\mathrm{Nb}$ atoms. The last structural model is a 15 atom model patterned on 6tria with each [111] B-site plane containing $\left[\mathrm{Zn}_{1 / 3} \mathrm{Nb}_{2 / 3}\right]$, which we name "3tria".

We begin by presenting the general features of the electronic structure of these 


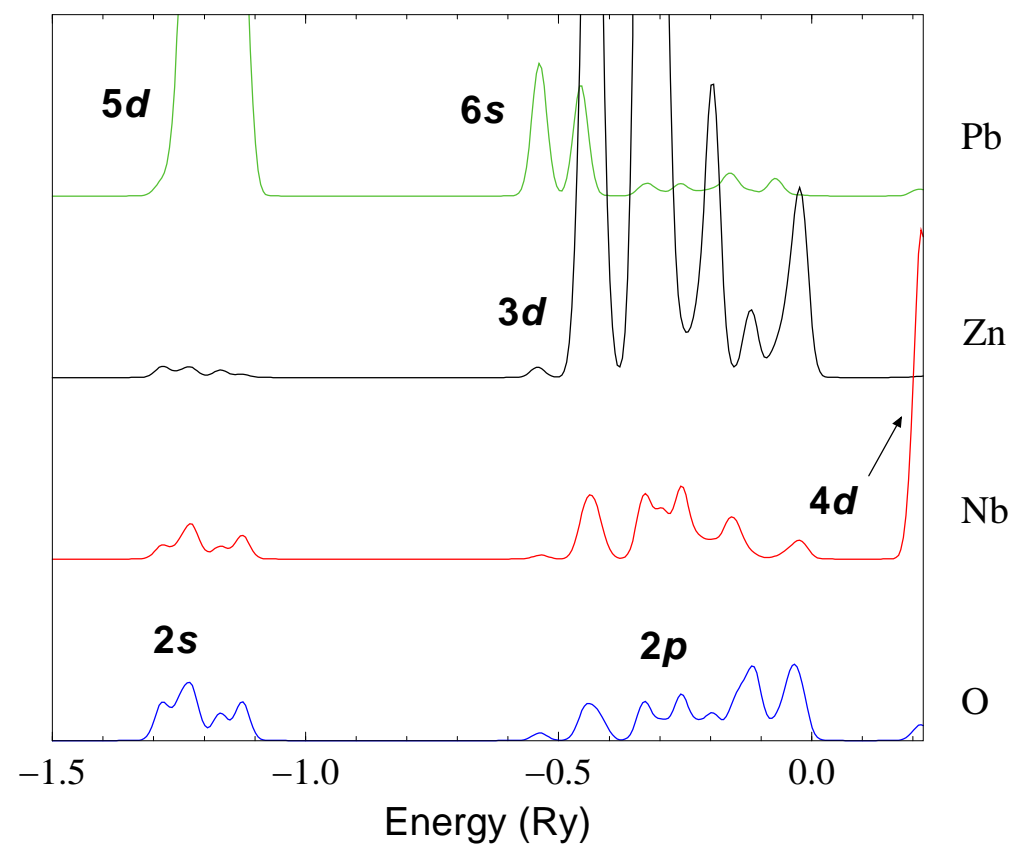

FIGURE 2. Density of states of 15 atom 3 -space-charge cell. The $s-p-d$ character of $\mathrm{Pb}, \mathrm{Zn}$, $\mathrm{Nb}$ and $\mathrm{O}$ were determined using sphere sizes of 2.0, 1.9, 1.6 and $1.65 \mathrm{bohr}$, respectively.

models. Figure 1 shows the band structure for 3spcchrg along high-symmetry directions in the Brillouin zone, referenced to the ideal $\mathrm{PbTiO}_{3}$ Brillouin zone, and Figure 2 shows the corresponding density of states projected onto the various atomic species. Strong Zn $3 d-\mathrm{O} 2 p$ bonding is evident in these figures. Other characteristic features of $\mathrm{PbTiO}_{3}$ [17], are also evident, such as strong $\mathrm{Nb} 4 d-\mathrm{O}$ $2 p$ bonding and $\mathrm{Pb} 6 s-\mathrm{O} 2 p$ bonding.

The distribution of nearest neighbor distances is presented in Figure 3 as pair distribution functions, artificially gaussian broadened. The dashed curves represent the ideal perovskite structure. There is large differences in the distribution of nearest neighbor distances in the different structures. The ranges of values are also tabulated in Table I. The characteristic ferroelectric splitting of Nb-O bondlengths is evident in all the structural models, with one bond shorter than ideal and the other longer. The widest variation occurs in the 6lin model. The $\mathrm{Zn}-\mathrm{O}$ distances are all closely clustered around the ideal value, the widest variation again occuring for the 6lin structure. By contrast with Nb-O, however, the Zn-O distances are mostly equal to or larger than the ideal perovskite B-O distance. By far the largest differences from ideal occur for the $\mathrm{Pb}-\mathrm{O}$ bond lengths. The widest variation occurs for the 3tria structure. In all cases, the lower end of the range is less than the ideal value, while the upper end is larger than ideal. Interestingly, the smallest variation occurs for the lowest energy 6tria structure. The ranges of values for the B-O distances agree well with the XAFS measurements of Chen et al. [18] for PZN, but less well for the $\mathrm{Pb}-\mathrm{O}$ bond length (only the shortest measured $\mathrm{Pb}-\mathrm{O}$ bond was 


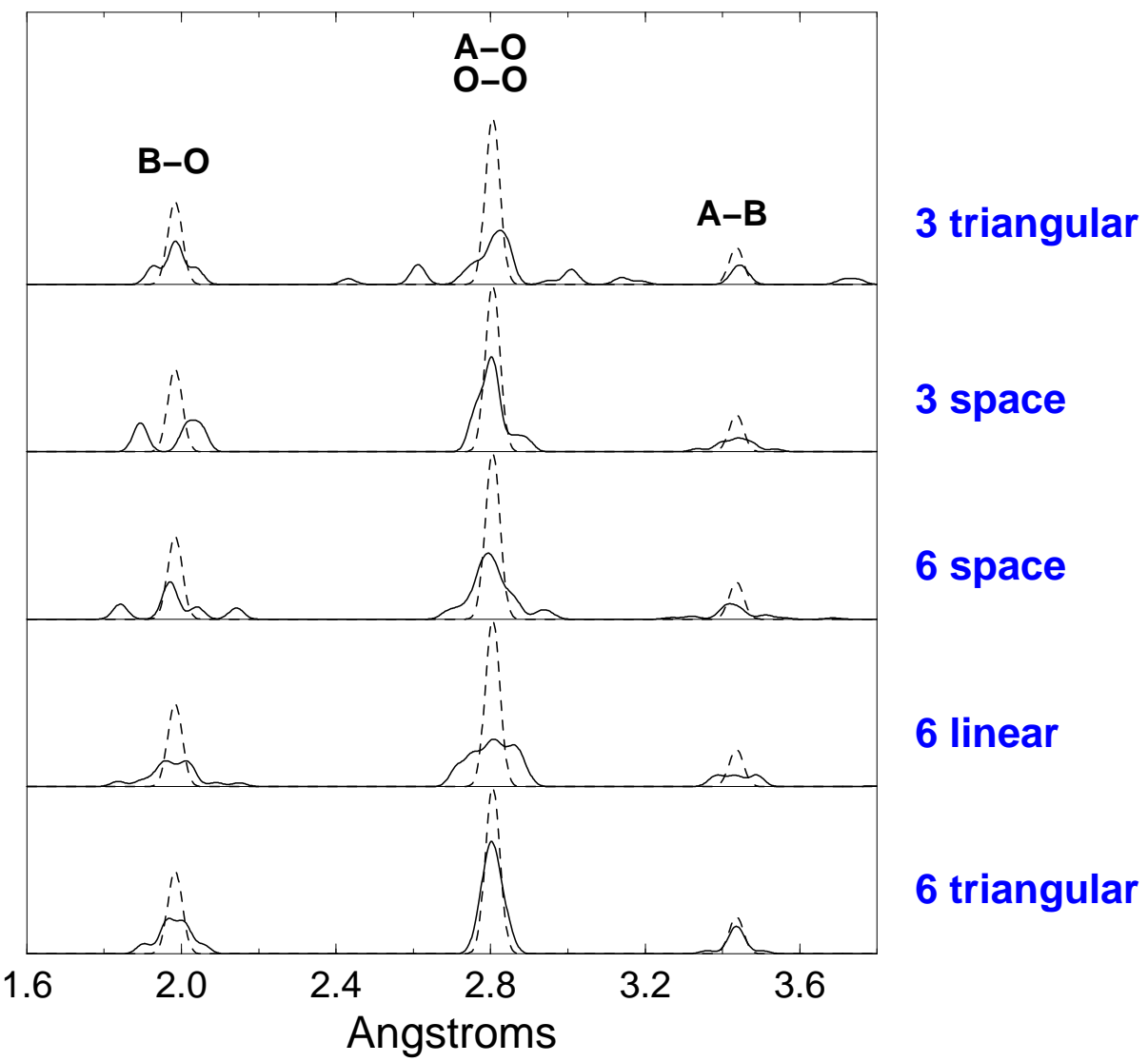

FIGURE 3. Pair distribution functions for relaxed and unrelaxed PZN structures

\begin{tabular}{|c|c|c|c|c|}
\hline & \multicolumn{3}{|c|}{ Distances $(\AA)$} & \multirow{2}{*}{$\begin{array}{c}\mathrm{E}_{T} \\
(\mathrm{mRy})\end{array}$} \\
\hline & $\mathrm{Pb}-\mathrm{O}$ & $\mathrm{Nb}-\mathrm{O}$ & $\mathrm{Zn}-\mathrm{O}$ & \\
\hline 6-triangle & $2.79-2.83$ & $1.90-2.06$ & 2.01 & 0 \\
\hline 3-triangle & $2.43-3.19$ & $1.93-1.98$ & $1.98-2.04$ & +30 \\
\hline 3-space & $2.76-2.86$ & $1.89-2.05$ & 2.02 & +39 \\
\hline 6-space & $2.69-2.95$ & $1.84-2.14$ & $1.98-2.04$ & +63 \\
\hline 6-linear & $2.71-2.87$ & $1.84-2.15$ & $2.01-2.09$ & +73 \\
\hline Ideal & 2.81 & 1.98 & 1.98 & - \\
\hline Expr. $^{\dagger}$ & $2.38,2.40^{*}$ & $1.92-2.12$ & 2.03 & - \\
\hline
\end{tabular}

${ }^{\top}$ I-Wei Chen, et al., 1995

*Egami et al., 1995

TABLE 1. Summary of PZN bond-lengths and total energies 
reported). The exception is the 3 -tria model, whose smallest value is $2.43 \AA$.

The total energies (per 15 atom PZN formula unit) of each of the five structures are compared in Table I, with all energies referenced to the 6tria model, which had the lowest total energy. The next most stable structures are the 3tria and $3 \mathrm{spc}$ models, with the 6lin and 6spc being least stable. This suggests that the random-site model (in the 6tria form) has the lowest energy. However, we must still investigate the effect of increasing the k-point sampling and reducing the imposed symmetry.

\section{CONCLUSIONS}

We have performed first-principles total energy and force studies of models of the solid solution relaxor ferroelectric PZN. Five different models were investigated incorporating the effects of [111] B-site ordering in both the space charge and random-site models. In all the calculations sizeable $\mathrm{Z}-\mathrm{O}$ bonding occurs, but the

$\mathrm{Z}-\mathrm{O}$ bond distances are all close to the ideal perovskite value. Comparisons of fully relaxed total energies in these initial calculations suggest that the random site model has the lowest energy. The effects of denser k-point sampling and reducing the imposed symmetry have yet to be investigated, however. $\mathrm{Nb}-\mathrm{O}$ and $\mathrm{Zn}-\mathrm{O}$ distances are consistent with XAFS measurements but the measured $\mathrm{Pb}-\mathrm{O}$ bond length is significantly smaller than all but one of the models. Detailed comparisons with neutron pair-distribution measurements would be desirable.

\section{REFERENCES}

1. S.-E. Park and T. R. Shrout, Journal of Materials Research Innovations, 1997, in press.

2. M. A. Akbas and P. K. Davies, preprint.

3. S. Teslic, T. Egami, and D. Viehland, Ferroelectrics 194, 271 (1997).

4. L. E. Cross, Ferroelectrics 151, 305 (1994); ibid. 76, 241 (1987).

5. H. B. Krause, J. M. Cowley, and J. Wheatley, Acta. Cryst. A35, 1015 (1979).

6. A. D. Hilton, C. A. Randall, D. J. Barber, and T. Shrout, Ferroelectrics 93, 379 (1988).

7. H. D. Rosenfeld and T. Egami, Ferroelectrics 164, 133 (1995).

8. D. Viehland and J. -F. Li, J. Appl. Phys. 74, 4121 (1993).

9. For a recent discussion of possible nanometer scale concentration inhomogeneities arising from Coulombic constraints see for example Refs. [1, 7] and A. G. Khachaturyan, Phys. Rev. B 48, 2949 (1993) and references therein.

10. R. Laiho, S. Lushnikov, and I. Siny, Ferroelectrics 125, 493 (1992).

11. D. A. Sagala and S. Nambu, J. Am. Ceram. Soc. 75, 2573 (1992).

12. D. J. Singh, H. Krakauer, C. Haas, and A. Y. Liu, Phys. Rev. B 46, 13065 (1992)

13. D. J. Singh, H. Krakauer, C. Haas, and W. E. Pickett, Nature 365, 39 (1993). 
14. K. M. Rabe and U. V. Waghmare, Phys. Rev. B 52, 13236 (1995), and references therein.

15. H. Krakauer, R. Yu, C.-Z. Wang, and C. LaSota, Ferroelectrics, in press, 1997; H. Krakauer, R. Yu, C.-Z. Wang, K. Rabe, and U. Waghmare, submitted; and references therein.

16. Y Liu, D. J. Singh, and H. Krakauer, Phys. Rev. B 49, 17424 (1994).

17. R. E. Cohen and H. Krakauer, Ferroelectrics 136, 65 (1992).

18. I-w. Chen, P. Li and Y. Wang, J. Phys. Chem Solids 57, 1525 (1996). 